\title{
Tensor microwave anisotropies from a stochastic magnetic field
}

\author{
R. Durrer \\ Département de Physique Théorique, Université de Genève, 24 quai Ernest Ansermet, CH-1211 Genève 4, Switzerland \\ P. G. Ferreira \\ Département de Physique Théorique, Université de Genève, 24 quai Ernest Ansermet, CH-1211 Genève 4, Switzerland; \\ CERN Theory Division, CH-1211, Geneve 23, Switzerland; \\ and CENTRA, Instituto Superior Técnico, Av. Rovisco Pais, 1, 1096 Lisboa Codex, Portugal \\ T. Kahniashvili \\ Department of Astrophysics, Abastumani Astrophysical Observatory, Kazbegi Ave. 2a, 380060 Tbilisi, Georgia \\ (Received 19 March 1999; revised manuscript received 13 October 1999; published 24 January 2000)
}

\begin{abstract}
We derive an expression for the angular power spectrum of cosmic microwave background anisotropies due to gravity waves generated by a stochastic magnetic field and compare the result with current observations; we take into account the non-linear nature of the stress energy tensor of the magnetic field. For almost scale invariant spectra, the amplitude of the magnetic field at galactic scales is constrained to be of order $10^{-9} \mathrm{G}$. If we assume that the magnetic field is damped below the Alfvén damping scale, we find that its amplitude at $0.1 h^{-1} \mathrm{Mpc}, B_{\lambda}$, is constrained to be $B_{\lambda}<7.9 \times 10^{-6} e^{3 n} \mathrm{G}$, for $n<-3 / 2$, and $B_{\lambda}<9.5 \times 10^{-8} e^{0.37 n} \mathrm{G}$, for $n>-3 / 2$, where $n$ is the spectral index of the magnetic field and $H_{0}=100 \mathrm{hm} \mathrm{s}^{-1} \mathrm{Mpc}^{-1}$ is the Hubble constant today.
\end{abstract}

PACS number(s): 98.62.En, 98.70.Vc, 98.80.Cq

\section{INTRODUCTION}

The past few years have seen a tremendous surge of interest in the origin and evolution of galactic magnetic fields [1]. A number of mechanisms have been proposed for the origin of the seed fields, ranging from inflationary mechanisms [2], cosmological phase transitions [3] to astrophysical processes [4]. Much progress has been made in trying to disentangle the various non-linear processes which may be responsible for the growth of such a seed field in the very early universe, in particular the interplay between the magnetic field and the primordial plasma $[5,6]$ and the importance of turbulence [7].

Given a small seed field at late times, two different mechanisms can cause its amplification to magnetic fields of order $10^{-6} \mathrm{G}$ observed in galaxies: adiabatic compression of magnetic flux lines can amplify a seed field of order $10^{-9} \mathrm{G}$ to the present, observable values; the far more efficient (and controversial) galactic dynamo mechanism may be able to amplify seed fields as small as $10^{-20} \mathrm{G}$ [4] or even $10^{-30} \mathrm{G}$ in universe with low mass density [8]. Clearly, to make some progress in identifying which one of these mechanisms is responsible for galactic magnetic fields, one would like to find a constraint for the seed field before it has been processed by local, galactic dynamics.

The obvious observable for such a constraint is the cosmic microwave background (CMB). It is interesting to note that a field strength of $10^{-8} \mathrm{G}$ provides an energy density of $\Omega_{B}=B^{2} /\left(8 \pi \rho_{c}\right) \sim 10^{-5} \Omega_{\gamma}$, where $\Omega_{\gamma}$ is the density parameter in photons. We naively expect a magnetic field of this amplitude to induce perturbations in the CMB on the order of $10^{-5}$, which are just on the level of the observed CMB anisotropies. It is thus justified to wonder to what extent the isotropy of the $\mathrm{CMB}$ may constrain primordial magnetic fields. Our order of magnitude estimate makes clear that we shall never be able to constrain tiny seed fields on the order of $10^{-13} \mathrm{G}$ or less in this way, but primordial fields of $10^{-9} \mathrm{G}$ may have left their traces in the CMB.

A number of methods have been proposed in the past few years for measuring a cosmological magnetic field using the CMB: the effect on the acoustic peaks [9], Faraday rotation on small [10] and large [11] scales and vorticity [12,13] can all lead to observable anisotropies in the CMB if the primordial magnetic field strength is of the order of $10^{-9}$ to $10^{-8} \mathrm{G}$. The most stringent bound from the CMB presented thus far was for the case of a homogeneous magnetic field [14]; the authors use the Cosmic Background Explorer (COBE) data to find the constraint $B_{0}<6.8$ $\times 10^{-9}\left(\Omega_{0} h^{2}\right)^{1 / 2} \mathrm{G}$ where the Hubble constant is $H_{0}$ $=100 \mathrm{~h} \mathrm{~km} \mathrm{~s}^{-1} \mathrm{Mpc}^{-1}$ and $\Omega_{0}$ is the energy density in units of the critical value. Although there is no fundamental reason to discard the possibility of a homogeneous magnetic field, all physical mechanisms proposed to date lead to the presence of stochastic magnetic fields with no homogeneous term; in this paper we consider such fields. For these types of configuration one is allowed to have fluctuations on a wide range of scales and the magnetic field will serve as a nonlinear driving force to the metric fluctuations; in the parlance of cosmological perturbation theory, the magnetic field evolves as a stiff source, without being affected by the fluid perturbations (back reaction) [15] which may be induced.

Stochastic magnetic fields have also been considered in [12], where the CMB anisotropies due to the induced fluid vorticity have been analyzed. Here we determine gravitational effects of the magnetic field. For simplicity, and to allow for a purely analytical analysis, we constrain ourselves to tensor perturbations. Similar contributions are also expected from vector and scalar perturbations which then 
would add to the final result. In this sense the anisotropies computed here are a strict lower bound (underestimating the true effect probably by about a factor of three).

The main result of this work is that one can obtain reasonably tight constrains for scale invariant magnetic fields; for causally generated magnetic fields the constraints are weaker and are strongly dependent on the evolution of the magnetic field in the radiation era on small scales.

For simplicity we concentrate on the case $\Omega_{0}=1$. Througout, we use conformal time which we denote by $\eta$. Greek indices run from 0 to 3 , Latin ones from 1 to 3 . We denote spatial (3D) vectors with bold face symbols. The value of the scale factor today is $a\left(\eta_{0}\right)=1$.

\section{THE STRESS TENSOR OF THE MAGNETIC FIELD}

During the evolution of the universe, the conductivity of the inter galactic medium is effectively infinite. In this regime we can decouple the time evolution from the spatial structure: $\mathbf{B}$ scales like $\mathbf{B}(\eta, \mathbf{x})=\mathbf{B}_{0}(\mathbf{x}) / a^{2}$ on sufficiently large scales. On smaller scales the interaction of the magnetic field with the cosmic plasma becomes important leading mainly to two effects: on intermediate scales, it oscillates like $\cos \left(\mathrm{v}_{A} k \eta\right)$, where $\mathrm{v}_{A}=B^{2} /[4 \pi(\rho+p)]^{1 / 2}$ is the Alfvén velocity and on small scales, the field is exponentially damped due to shear viscosity [6].

We will model $\mathbf{B}_{0}(\mathbf{x})$ as a statistically homogeneous and isotropic random field. The transversal nature of $\mathbf{B}$ leads us to

$$
\left\langle B_{i}(\mathbf{k}) B_{j}^{*}(\mathbf{q})\right\rangle=\delta^{3}(\mathbf{k}-\mathbf{q})\left(\delta_{i j}-\hat{k}_{i} \hat{k}_{j}\right) B^{2}(k),
$$

where we use the Fourier transform conventions

$$
\begin{aligned}
B_{j}(\mathbf{k}) & =\int d^{3} x \exp (i \mathbf{x} \cdot \mathbf{k}) B_{0 j}(\mathbf{x}) \\
B_{0 j}(\mathbf{x}) & =\frac{1}{(2 \pi)^{3}} \int d^{3} k \exp (-i \mathbf{x} \cdot \mathbf{k}) B_{j}(\mathbf{k}) .
\end{aligned}
$$

The Alfvén oscillations modulate the initial power spectrum by a factor

$$
B^{2}(k) \rightarrow B^{2}(k) \cos ^{2}\left(\mathrm{v}_{A} k \eta\right) .
$$

This can be approximated by a reduction of a factor 2 in the power spectrum on scales with $\vee_{A} k \eta \gtrsim 1$. But as we shall see, our most stringent constraints will come either from very small scales where the spectrum is exponentially damped or from much larger scales where oscillations can be ignored. We will incorporate the exponential damping by a cutoff in the power spectrum at the damping scale.
Let us investigate the consequence of causality for the spectrum $B^{2}(k)$. If $\mathbf{B}$ is generated by some causal mechanism, it is uncorrelated on super horizon scales:

$$
\left\langle B_{i}(\mathbf{x}, \eta) B_{j}\left(\mathbf{x}^{\prime}, \eta\right)\right\rangle=0 \quad \text { for } \quad\left|\mathbf{x}-\mathbf{x}^{\prime}\right|>2 \eta .
$$

Here it is important that the universe is in a stage of standard Friedmann expansion, so that the causal horizon size is about $\eta$. During an inflationay phase the causal horizon diverges and our subsequent argument does not apply. In this somewhat misleading sense, one calls inflationary perturbations "a-causal."

According to Eq. (2), $\left\langle B_{i}(\mathbf{x}, \eta) B_{j}\left(\mathbf{x}^{\prime}, \eta\right)\right\rangle$ is a function with compact support and hence its Fourier transform is analytic. The function

$$
\left\langle B_{i}(\mathbf{k}) B_{j}^{*}(\mathbf{k})\right\rangle \equiv\left(\delta_{i j}-\hat{k}_{i} \hat{k}_{j}\right) B^{2}(k)
$$

is analytic in $\mathbf{k}$. If we in addition assume that $B^{2}(k)$ can be approximated by a simple power law, we must conclude that $B^{2}(k) \propto k^{n}$, where $n \geqslant 2$ is an even integer. (A white noise spectrum, $n=0$ does not work because of the transversality condition which has led to the non-analytic pre-factor $\delta_{i j}$ $-\hat{k}_{i} \hat{k}_{j}$.) By causality, there can be no deviations of this law on scales larger than the horizon size at formation, $\eta_{i n}$.

We assume that the probability distribution function of $\mathbf{B}_{0}$ is Gaussian; although this is not the most general random field, it greatly simplifies calculations and gives us a good idea of what to expect in a more general case.

The anisotropic stresses induced are given by the convolution of the magnetic field,

$$
\tau_{i j}^{(B)}(\mathbf{k})=\frac{1}{4 \pi} \int d^{3} q B_{i}(\mathbf{q}) B_{j}^{*}(\mathbf{k}-\mathbf{q})-\frac{1}{2} B_{l}(\mathbf{q}) B_{l}^{*}(\mathbf{k}-\mathbf{q}) \delta_{i j} .
$$

With the use of the projection operator, $P_{i j}=\delta_{i j}-\hat{k}_{i} \hat{k}_{j}$ we can extract the tensor component of Eq. (4),

$$
\Pi_{i j}^{(B)}=\left[P_{i}^{a} P_{j}^{b}-(1 / 2) P_{i j} P^{a b}\right] \tau_{a b},
$$

tracelessness, orthogonality, and symmetry force the correlation function to be of the form

$$
\begin{aligned}
& \left\langle\Pi_{i j}^{(B)}(\mathbf{k}, t) \Pi_{l m}^{(B)} *\left(\mathbf{k}^{\prime}, t\right)\right\rangle=\left|\Pi_{B}(k, t)\right|^{2} \mathcal{M}_{i j l m} \delta\left(\mathbf{k}-\mathbf{k}^{\prime}\right) \\
& \left\langle\Pi_{i j}^{(B)}(\mathbf{k}, t) \Pi_{i j}^{(B)} *\left(\mathbf{k}^{\prime}, t\right)\right\rangle=4\left|\Pi_{B}(k, t)\right|^{2} \delta\left(\mathbf{k}-\mathbf{k}^{\prime}\right),
\end{aligned}
$$

where we make use of the tensor basis, $\mathcal{M}$ : The correlator of an isotropic tensor component has always the following tensorial structure:

$$
\mathcal{M}_{i j l m}=\delta_{i l} \delta_{j m}+\delta_{i m} \delta_{j l}-\delta_{i j} \delta_{l m}+k^{-2}\left(\delta_{i j} k_{l} k_{m}+\delta_{l m} k_{i} k_{j}-\delta_{i l} k_{j} k_{m}-\delta_{i m} k_{l} k_{j}-\delta_{j l} k_{i} k_{m}-\delta_{j m} k_{l} k_{i}\right)+k^{-4} k_{i} k_{j} k_{l} k_{m} .
$$

We now determine the function $\left|\Pi_{B}(k, t)\right|^{2}$ in terms of the magnetic field. Using Wick's theorem we have

$$
\left\langle B_{i}(\mathbf{k}) B_{j}^{*}(\mathbf{q}) B_{n}(\mathbf{s}) B_{m}^{*}(\mathbf{p})\right\rangle=\left\langle B_{i}(\mathbf{k}) B_{j}^{*}(\mathbf{q})\right\rangle\left\langle B_{n}(\mathbf{s}) B_{m}^{*}(\mathbf{p})\right\rangle+\left\langle B_{i}(\mathbf{k}) B_{n}^{*}(\mathbf{s})\right\rangle\left\langle B_{j}(\mathbf{q}) B_{m}^{*}(\mathbf{p})\right\rangle+\left\langle B_{i}(\mathbf{k}) B_{m}^{*}(\mathbf{p})\right\rangle\left\langle B_{n}(\mathbf{s}) B_{j}^{*}(\mathbf{q})\right\rangle .
$$


The problem reduces itself to calculating self convolutions of the magnetic field. The power spectrum of Eq. (4) is

$$
\begin{aligned}
\left\langle\tau_{i j}^{B}(\mathbf{k}, \eta) \tau_{l m}^{B *}\left(\mathbf{k}^{\prime}, \eta\right)\right\rangle= & \frac{1}{(8 \pi)^{2}} \int d^{3} q \int d^{3} p\left\langle B_{i}(\mathbf{q}) B_{j}((\mathbf{k}-\mathbf{q})) B_{l}(\mathbf{p}) B_{m}\left(\left(\mathbf{p}-\mathbf{k}^{\prime}\right)\right)\right\rangle \\
= & \delta\left(\mathbf{k}-\mathbf{k}^{\prime}\right) \int d^{3} q B^{2}(q) B^{2}(|\mathbf{k}-\mathbf{q}|) \times\left\{( \delta _ { i l } - \hat { q } _ { i } \hat { q } _ { l } ) \left[\delta_{j m}-\widehat{\left.(\mathbf{k}-\mathbf{q})_{j}(\widehat{\mathbf{k}-\mathbf{q}})_{m}\right]}\right.\right. \\
& \left.+\left(\delta_{i m}-\hat{q}_{i} \hat{q}_{m}\right)\left[\delta_{j l}-(\widehat{\mathbf{k}-\mathbf{q}})_{j}(\widehat{\mathbf{k}-\mathbf{q}})_{l}\right]\right\} .
\end{aligned}
$$

Using Eqs. (8), (5) and (6), this leads to $\left|\Pi_{B}\right|^{2}=f(k)^{2} / a^{8}$, where

$$
f(k)^{2}=\frac{1}{(8 \pi)^{2}} \int d^{3} q B^{2}(q) B^{2}(|\mathbf{k}-\mathbf{q}|)\left(1+2 \gamma^{2}+\gamma^{2} \beta^{2}\right),
$$

with $\gamma=\hat{\mathbf{k}} \cdot \hat{\mathbf{q}}$ and $\beta=\hat{\mathbf{k}} \cdot \widehat{\mathbf{k}-\mathbf{q}}$.

It remains to define $B(k)$ from Eq. (1). We shall parametrize it in terms of an amplitude and a scale dependence through

$$
B^{2}(k)= \begin{cases}\frac{(2 \pi)^{5}}{4} \frac{\lambda^{n+3}}{\Gamma[(n+3) / 2]} B_{\lambda}^{2} k^{n} & \text { for } k<k_{c}, \\ 0 & \text { otherwise. }\end{cases}
$$

The normalization is such that $\left.\left\langle B_{0}^{i}(\mathbf{x}) B_{0}^{i}(\mathbf{x})\right\rangle\right|_{\lambda}=B_{\lambda}^{2}$ where the quantity in brackets represents the averaged magnetic field smoothed over a comoving length scale $\lambda$. Note that we have assumed that the cutoff scale today is smaller than $\lambda$.

We require $n \geqslant 3$ so as not to over-produce long range coherent fields; we shall see that for $n=-3$ we obtain a scale invariant spectrum of CMB anisotropies.

We have included a short wavelength cutoff to take into account the exponential damping due to shear viscosity in the cosmic plasma [6]. The mean energy density due to such a magnetic field, which is an appropriately weighted integral of Eq. (11), will be strongly dependent on the cutoff when $n>-3$.

Using Eqs. (11) and (10) we can calculate $f$. The integral cannot be computed analytically, but the following result is a good approximation for all wave numbers $k$ :

$$
\begin{aligned}
f^{2}(k) \simeq & \frac{(2 \pi)^{9}}{16} \frac{\lambda^{2 n+6} B_{\lambda}^{4}}{\Gamma^{2}[(n+3) / 2](2 n+3)} \\
& \times\left(k_{c}^{2 n+3}+\frac{n}{n+3} k^{2 n+3}\right) .
\end{aligned}
$$

This result seems to have a singularity at $n=-3 / 2$ which is however removable. The first term dominates if $n>-3 / 2$ and the second term dominates if the opposite inequality is satisfied. For $n>-3 / 2$, the gravity wave source is therefore white noise and its amplitude is determined by the upper cutoff, $k_{c}$. Note that if $n>-3 / 2$, the spectrum of the energy momentum tensor becomes white noise, independent of $n$.
Only the amplitude which is proportional to $\left(\lambda k_{c}\right)^{2 n}$ depends on the spectral index. This is due to the fact that the integral (10) is dominated by the contributions at very small scales, $k_{c} \gg k$. The induced $C_{l}$ spectrum from gravity wave will therefore be independent of $n$ for $n>-3 / 2$, and obey the well known behavior $C_{l} \propto l$ of a white noise source.

To simplify, we just consider the dominant term and, in order not to artificially produce a singularity at $n=-3 / 2$, we drop the factor $1 /(2 n+3)$. Given the intent of this paper (to constrain the amplitude of the magnetic field) we will include a factor of $10^{-1}$ in our final result, guaranteeing that we are not overestimating $\mathrm{CMB}$ anisotropies. The singularity at $n=-3$ is real. It is the usual logarithmic singularity of the scale invariant spectrum.

\section{THE CMB ANISOTROPIES}

Armed with the structure and evolution of the stochastic magnetic field we can now proceed to calculate its effects on tensor CMB anisotropies. The metric element of the perturbed Friedman universe is given by

$$
d s^{2}=a^{2}(\eta)\left[-d \eta^{2}+\left(\delta_{i j}+2 h_{i j}\right) d x^{i} d x^{j}\right],
$$

where $h_{i}^{i}=0$ and $h_{i}^{j} k^{i}=0$ for tensor perturbations [16]. The magnetic field will source the evolution equation for $h_{i j}$ through

$$
\ddot{h}_{i j}+2 \frac{\dot{a}}{a} \dot{h}_{i j}+k^{2} h_{i j}=8 \pi G \Pi_{i j}^{(B)} .
$$

Such a gravity wave induces temperature fluctuations in the CMB due to the fact that the photons move along the perturbed geodesics [15]

$$
\frac{\Delta T}{T}\left(\eta_{0}, \mathbf{x}, \mathbf{n}\right)=\int_{\eta_{*}}^{\eta_{0}} \dot{h}_{i j}(\mathbf{x}(\eta), \eta) n^{i} n^{j} d \eta .
$$

Here $\eta_{*}$ denotes the (conformal) time of decoupling of matter and radiation due to recombination. We want to compute the angular power spectrum of $\Delta T / T$, the $C_{l}$, defined by

$$
\left\langle\frac{\Delta T}{T}(\mathbf{n}) \frac{\Delta T}{T}\left(\mathbf{n}^{\prime}\right)\right\rangle_{\mathbf{n} \cdot \mathbf{n}^{\prime}=\mu}=\frac{1}{4 \pi} \sum_{l}(2 l+1) C_{l} P_{l}(\mu) .
$$

The $C_{l}$ s are solely determined by the power spectrum of metric fluctuations. Defining 


$$
\left\langle\dot{h}_{i j}^{(T)}\left(\mathbf{k}^{\prime}, \eta\right) \dot{h}_{l m}^{(T) *}(\mathbf{k}, \eta)\right\rangle=|\dot{H}(k, \eta)|^{2} \mathcal{M}_{i j l m} \delta\left(\mathbf{k}-\mathbf{k}^{\prime}\right)
$$

one can derive a closed form expression for $C_{l}$ (see [17]):

$$
\begin{aligned}
C_{l} & =\frac{1}{4 \pi^{4}} \int d k k^{2}|I(l, k)|^{2} l(l-1)(l+1)(l+2), \\
I & =\int_{\eta_{*}}^{\eta_{0}} d \eta \dot{H}(\eta, k)\left(\frac{j_{l}\left(k\left(\eta_{0}-\eta\right)\right)}{\left(k\left(\eta_{0}-\eta\right)\right)^{2}}\right),
\end{aligned}
$$

where $j_{l}$ denotes the spherical Bessel function of order $l$. We solve Eq. (13) using the Wronskian method; in terms of the dimensionless variable $x=k \eta$. The homogeneous solutions are the spherical Bessel functions $j_{0}, y_{0}$ in the radiation dominated era, and $j_{1} / x, y_{1} / x$ in the matter dominated era, respectively. We assume that the magnetic fields were created in the radiation dominated epoch, at redshift $z_{i n}$. We then match the general inhomogeneous solutions of Eq. (13) at the time of equal matter and radiation, $\eta_{e q}$. Because of the rapid falloff of the source term in the matter dominated era, the perturbations created after $\eta_{e q}$ are sub-dominant, and we find, for the dominant contribution at $\eta>\eta_{e q}$,

$$
\dot{H}(k, t) \simeq 4 \pi G \eta_{0}^{2} z_{e q} \ln \left(\frac{z_{\text {in }}}{z_{e q}}\right) k f(k) \frac{j_{2}(k \eta)}{k \eta} .
$$

Inserting this result in Eq. (16), we obtain

$$
I=4 \pi G \eta_{0}^{2} z_{e q} \ln \left(z_{i n} / z_{e q}\right) f(k) \int_{x_{*}}^{x_{0}} d x \frac{j_{2}(x)}{x} \frac{j_{l}\left(x_{0}-x\right)}{\left(x_{0}-x\right)^{2}},
$$

where $x=k \eta, x_{*}=k \eta_{*}$ and $x_{0}=k \eta_{0}$. For wave numbers which are super-horizon at decoupling, $x_{*}<\pi$, the lower boundary in Eq. (18) can be set to 0. The remaining integral cannot be expressed in closed form, but is well approximated by $[20]$

$$
\begin{aligned}
\int_{0}^{x_{0}} d x \frac{j_{2}(x)}{x} \frac{j_{l}\left(x_{0}-x\right)}{\left(x_{0}-x\right)^{2}} & =\frac{\pi}{2} \int_{0}^{x_{0}} d x \frac{J_{5 / 2}(x)}{x^{3 / 2}} \frac{J_{l+1 / 2}\left(x_{0}-x\right)}{\left(x_{0}-x\right)^{5 / 2}} \\
& \simeq \frac{0.7 \pi}{2} \int_{0}^{x_{0}} d x \frac{J_{5 / 2}(x)}{x} \frac{J_{l+1 / 2}\left(x_{0}-x\right)}{\left(x_{0}-x\right)^{3}} \\
& =\frac{7 \pi}{25} \sqrt{\frac{l}{x_{0}^{3}}} J_{l+3}\left(x_{0}\right) .
\end{aligned}
$$

The third integral above can be expressed in closed form ([21], number 6.581.2), and is reasonably well approximated by the last expression, we have checked the approximation numerically for $l \leqslant 200$ and varying $x_{0}$.

We can now do the integrations in Eq. (15) analytically to obtain

$$
l^{2} C_{l} \simeq A\left(\frac{\lambda}{\eta_{0}}\right)^{2 n+6} \frac{2}{3 \pi}\left(k_{c} \eta_{0}\right)^{2 n+3} l^{3}
$$

for $n>-3 / 2$, and

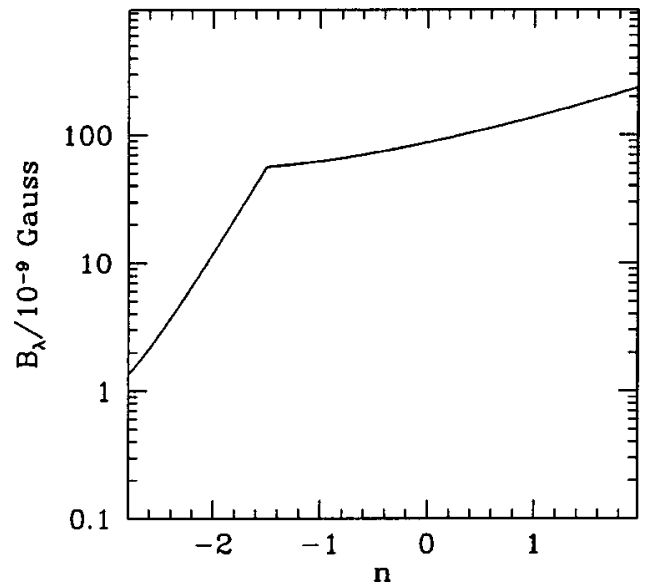

FIG. 1. The upper bound $B_{\lambda}$ as a function of spectral index, $n$. We assume $z_{\text {in }} / z_{\text {eq }}=10^{9}$ and $\lambda=0.1 h^{-1} \mathrm{Mpc}$.

$$
l^{2} C_{l} \simeq A\left(\frac{\lambda}{\eta_{0}}\right)^{2 n+6} \frac{-n}{(n+3)} \frac{\Gamma[1-2 n]}{\Gamma^{2}[1-n] 2^{(1-2 n)}} l^{6+2 n}
$$

for $-3<n<-3 / 2$, where

$$
\begin{aligned}
A & =5 \times 10^{-4}(2 \pi)^{9} z_{e q}^{2} \ln ^{2}\left(\frac{z_{\text {in }}}{z_{e q}}\right) \frac{B_{\lambda}^{4} G^{2} \eta_{0}^{4}}{\Gamma^{2}[(n+3) / 2]} \\
& =310^{-8}\left(\frac{B_{\lambda}}{10^{-9} \mathrm{G}}\right)^{4} \ln ^{2}\left(z_{\text {in }} / z_{\text {eq }}\right) \frac{1}{\Gamma^{2}[(n+3) / 2]} .
\end{aligned}
$$

\section{RESULTS}

Equations (20) and (21) are our main result. They allow us to limit a possible primordial magnetic field by requiring it not to over produce fluctuations in the CMB. Since the fluctuations induced grow with $l$ for all values of the spectral index $-3<n$, we obtain the best limits for large values of $l$. We shall be conservative and assume an upper bound of $\left.l^{2} C_{l}\right|_{l=50}<8.5 \times 10^{-9}$ [18]. Given that we are interested in galactic and cluster scales we fix $\lambda=0.1 h^{-1} \mathrm{Mpc}$ for the remainder of this paper. In Fig. 1 we show the limit on a stochastic magnetic field as a function of the spectral index $n$, using the damping scale given below as cutoff.

We now focus on a few particular cases of interest and in doing so we will derive an analytic expression which approximates the upper bound of $B_{\lambda}$ over the whole range of $n$.

Scale invariant magnetic field: From Eq. (21) we see that the result is independent of the cutoff. In the limit where $n$ $\rightarrow-3$ we find that

$$
B_{\lambda} \lesssim 10^{-9} \mathrm{G}
$$

i.e., of the same level as other constraints [9-14].

Causal magnetic field: For this scenario we have, as explained above, $n \geqslant 2$; we shall consider the case of $n=2$. For instructive purposes let us first consider a $k_{c}$ which is independent of the magnetic field. The constraint is then 


$$
B_{\lambda} \lesssim \ln ^{-1 / 2}\left(\frac{z_{\text {in }}}{z_{\text {eq }}}\right)\left(k_{c} \eta_{0}\right)^{-7 / 4} \mathrm{G} .
$$

The cutoff $k_{c}$ will depend on the plasma properties and evolution; even though the conductivity $\sigma$ of the cosmic plasma is very large, it is nevertheless finite. One actually finds [19] that $\sigma=\alpha T$, where the parameter $1<\alpha<7$ is slowly temperature dependent. By Ohm's law, magnetic fields on small enough scales are exponentially suppressed, $B \propto \exp \left(-a k^{2} \eta / 4 \pi \sigma\right)$, leading to a damping scale, $k_{d}(\eta)$ $=(4 \pi \sigma a / \eta)^{1 / 2}=\left(\eta \times 2 \times 10^{-3} \mathrm{~cm}\right)^{-1 / 2}$. This scale is smaller than the comoving horizon scale for all temperatures below the Planck scale. On scales smaller than $1 / k_{d}\left(\eta_{e q}\right)$, the induced gravity waves have damped away even before matter and radiation equality. Since the sourcing of gravity waves after equality is negligible, the damping scale relevant in our problem is $k_{c}=k_{d}\left(\eta_{e q}\right)$,

$$
k_{c} \simeq 2 \times 10^{13} h^{2} \mathrm{Mpc}^{-1} .
$$

If we insert this damping scale in Eq. (24), we obtain $B_{\lambda}$ $\lesssim 10^{-29} \mathrm{G}$.

A more realistic scenario is to assume that the magnetic field will be damped by electron viscosity. To proceed with the analysis we shall split the stochastic magnetic field into a high-frequency component and a low-frequency component; the scale which separates the two is the Alfven scale at equality, $\lambda_{A}=V_{A} \eta_{e q}$ where $V_{A}$ is the Alfven velocity, $V_{A}^{2}$ $=\left\langle B^{2}\right\rangle /(4 \pi(\rho+p))$. From Eq. (4) of [12] we see that the inhomogeneous magnetic field will obey a damped harmonic oscillator equation, with a time dependent damping coefficient, $D=0.2 k^{2} l_{\gamma}(1+z)\left(l_{\gamma}\right.$ is the physical photon mean free path) and frequency $\omega^{2}(\eta)=V_{A}^{2} k^{2}-(\dot{D} / 2)-(D / 2)^{2}$. Within this setting we can estimate the damping scale of the magnetic field in the oscillatory regime of this system; the amplitude of the effective homogeneous magnetic field, $B_{A}$, which is responsible for the Alfvén waves is related to $B_{\lambda}$ through

$$
B_{A}^{2} \simeq B_{\lambda}^{2}\left(\frac{\lambda 10^{-9} \mathrm{G}}{3.8 \times 10^{-4} B_{A} \eta_{e q}}\right)^{n+3}
$$

which leads to

$$
B_{A}=\left(\frac{B_{\lambda}}{10^{-9} \mathrm{G}}\right)^{2 /(n+5)}\left(13 h^{-1}\right)^{(n+3) /(n+5)} 10^{-9} \mathrm{G} .
$$

We shall define the damping scale to be the scale at which one e-fold of damping has occured by equality. From $\int_{0}^{\eta_{e q}}(D / 2) d \eta=1$ one finds

$$
k_{c}=4.5 \mathrm{Mpc}^{-1} .
$$

For this estimate to be valid, the system must be in the damped oscillatory regime (as opposed to overdamping regime), i.e., $\omega^{2}\left(\eta_{e q}\right)>0$; this condition is satisfied if $B_{A}$ $>5.5 h^{-2} \times 10^{-9} \mathrm{G}$. We find that indeed this is the case in the range of interest.
Combining Eqs. (20), (21), and (27) and assuming a formation redshift of $z_{i n}=10^{15}$ (although the final result is very weakly dependent $z_{i n}$ ) we find that an approximation to the bound is

$$
\begin{aligned}
& B_{\lambda}<7.9 \times 10^{-6} e^{2.99 n} \mathrm{G}, \quad \text { for } n<-3 / 2, \\
& B_{\lambda}<9.5 \times 10^{-8} e^{0.37 n} \mathrm{G}, \quad \text { for } n>-3 / 2 .
\end{aligned}
$$

The upper bounds corresponding to Eq. (28) represent a reasonable fit to Fig. 1. As one can see, the constraint on a causal magnetic field is well above $10^{-9} \mathrm{G}$.

Throughout this derivation we have assumed that we can estimate the damping scale of the magnetic field by looking solely at the Alfven modes. A linear analysis of the remaining degrees of freedom also indicates that the magnetic field will be damped at the same scale as in Eq. (27). It is possible that nonlinear effects may prevent the tangled magnetic field from damping at this scale but an accurate quantitative analysis is still lacking.

Inflationary magnetic fields: Broken conformal invariance allied with the inflationary period will create large scale magnetic fields. Bertolami and Mota [2] estimate the spectral index in such a mechanism to lie in the range around 0 . We are then clearly in the regime where the cutoff is important. Using the Alfvén damping scale at equality and assuming magnetic field generation at $10^{10} \mathrm{GeV}$, we find $B_{\lambda}$ $\lesssim\left(10^{-7}-10^{-8}\right) \quad \mathrm{G}$ for $n$ varying from -0.5 to 0.5 . A similar result can be obtained for the model of Gasperini et al. [2].

\section{DISCUSSION}

Our calculation differs from most of the recent work on the impact of primordial magnetic fields on structure formation: In estimating the $\mathrm{CMB}$ anisotropies we do not split the magnetic field into a "large" homogenous mode and a "small" fluctuation. The magnetic field then affects metric perturbations quadratically. This has two effects. First it allows us to consider the magnetic field as a stiff source, and discard [within the magneto hydrodynamics (MHD) approximation] the back reaction of the perturbations in the cosmological fluid. Indeed if we were to consider back reaction then we would know a priori that we would be generating unacceptable perturbations in the cosmological fluid. Another way of phrasing this is that the magnetic field itself is $1 / 2$ order perturbation theory, while its energy momentum tensor and consequently the induced metric perturbations are first order perturbations. The MHD back reactions on B would be $3 / 2$ order and may thus be neglected in linear perturbation theory. We point out, however that, to obtain an estimate of the damping scale due to the viscosity in the MHD we had to consider a split between long wave length and short wavelength fluctuations in $\mathbf{B}$.

Second, the stress energy tensor being quadratic in the magnetic field, leads to a "sweeping" of modes: large wavelength modes in $T_{\mu \nu}$ will in general be affected by all scales of the spectrum of $B$ [12]. As we have seen in the causal case, the small wavelength behavior of the magnetic field totally dominates the large wavelength pertubations. In [5] 
the magnetic field is modeled as $\mathbf{B}=\overline{\mathbf{B}}+\mathbf{B}^{(1)}(\mathbf{x})$ where $\overline{\mathbf{B}}$ is a homogeneous term; the stress energy tensor is then given by terms of the form $\bar{B}_{i} B_{j}^{(1)}$, which are linear in the stochastic component.

A few comments are in order with regards to our result. Note that we are considering a specific class of models, where the magnetic field seed is created at some well defined moment in the early universe and then evolves according to the MHD equations. If the magnetic field is being constantly sourced throughout the radiation era, then our calculation is not valid. An example of such a scenario was proposed by Vachaspati [3] where magentic fields are sourced by vortical imprints from an evolving network of cosmic strings; although the scaling behavior of source may lead to $B \propto a^{-2}$, the effective damping scale will be of order the horizon much larger than the Alfvén damping scale. Another possibility has been put forward in [7], where the onset of turbulence induces an amplification of power on large scales but a supression of power on small scales. This would further increase $k_{c}$ but the results are still too qualitative to be properly included in an analysis such as ours.

\section{ACKNOWLEDGMENTS}

We thank John Barrow, Orfeu Bertolami, Kari Enqvist, Karsten Jedamzik, João Magueijo, Evan Scannapiecco and Misha Shaposhnikov for useful discussions. R.D. acknowledges the hospitality of the CfPA at U.C. Berkeley, where this work was initiated. T.K. acknowledges the hospitality of Geneva University.
[1] P. P. Kronberg, Rep. Prog. Phys. 57, 57 (1994).

[2] M. S. Turner and L. M. Widrow, Phys. Rev. D 37, 2743 (1988); B. Ratra, Astrophys. J. Lett. 391, L1 (1992); W. D. Garretson, G. B. Field, and S. M. Carroll, Phys. Rev. D 46, 5346 (1992); M. Gasperini, M. Giovannini, and G. Veneziano, Phys. Rev. Lett. 75, 3796 (1995); D. Lemoine and M. Lemoine, Phys. Rev. D 52, 1955 (1995); O. Bertolami and D. F. Mota, gr-qc/9901041.

[3] T. W. B. Kibble and A. Vilenkin, Phys. Rev. D 52, 679 (1995); J. T. Ahonen and K. Enqvist, ibid. 57, 664 (1998); T. Vachaspati, Phys. Lett. B 265, 258 (1991); M. Joyce and M. E. Shaposhnikov, Phys. Rev. Lett. 79, 1193 (1997).

[4] Ya. B. Zeldovich, A. A. Ruzmaikin, and D. D. Sokoloff, Magnetic Fields in Astrophysics (Gordon and Breach, New York, 1983); E. N. Parker, Cosmological Magnetic Fields (Oxford University Press, Oxford, England, 1979).

[5] E. Kim, A. Olinto, and R. Rosner, Astrophys. J. 468, 28 (1996); K. Jedamzik, V. Katalinic, and A. Olinto, Phys. Rev. D 57, 3264 (1998).

[6] K. Subramanian and J. Barrow, Phys. Rev. D 58, 083502 (1998).

[7] A. Brandenburg, K. Enqvist, and P Oleson, Phys. Rev. D 54, 1291 (1996).

[8] A. Davis, M. Lilley, and O. Tornqvist, Phys. Rev. D 60, 021301 (1999).
[9] J. Adams, U. H. Danielsson, D. Grasso, and H. Rubinstein, Phys. Lett. B 388, 253 (1996).

[10] A. Kosowsky and A. Loeb, Astrophys. J. 469, 1 (1996).

[11] E. Scannapieco and P. G. Ferreira, Phys. Rev. D 56, R7493 (1997).

[12] K. Subramanian and J. Barrow, Phys. Rev. Lett. 81, 3575 (1998).

[13] R. Durrer, T. Kahniashvili, and A. Yates, Phys. Rev. D 58, 123004 (1998).

[14] J. Barrow, P. Ferreira, and J. Silk, Phys. Rev. Lett. 78, 3610 (1997).

[15] R. Durrer, Fundam. Cosm. Phys. 15, 209 (1994).

[16] P. J. Peebles, The Large Scale Structure of the Universe (Princeton University Press, Princeton, NJ, 1980).

[17] F. F. Abott and M. B. Wise, Nucl. Phys. B244, 541 (1984).

[18] J. Ruhl et al., Astrophys. J. Lett. 453, L1 (1995).

[19] This formula for the conductivity has been derived in $\mathrm{J}$. Ahonen and K. Enqvist, Phys. Lett. B 382, 40 (1996) for $T$ $\gtrsim 1 \mathrm{MeV}$. For $T<1 \mathrm{MeV}$, the scattering processes which determine the conductivity are Compton scattering and Rutherford scattering. Applying a simple formula for the conductivity in a non-relativistic plasma, one obtains the same result with $\alpha \sim 4$.

[20] M. Abramowitz and I. Stegun, Handbook of Mathematical Functions (Dover, New York, 1972).

[21] Tables of Integrals, Series and Products (Academic, New York, 1993). 